\section{0}

CHOICE

Vanderbilt University Medical Center, Nashville, Tennessee, USA

\section{Correspondence to}

Dr John E Kuhn, Chief of Shoulder Surgery, Vanderbilt University Medical Center, Vanderbilt Sports Medicine, 3200 MCE South Tower, 1215 21st Avenue South, Nashville, TN 37232,USA; j.kuhn@ vanderbilt.edu

Accepted 31 December 2009

\title{
A new classification system for shoulder instability
}

\author{
John E Kuhn
}

\section{ABSTRACT}

Glenohumeral joint instability is extremely common yet the definition and classification of instability remains unclear. In order to find the best ways to treat instability, the condition must be clearly defined and classified. This is particularly important so that treatment studies can be compared or combined, which can only be done if the patient population under study is the same. The purpose of this paper was to review the problems with historical methods of defining and classifying instability and to introduce the FEDS system of classifying instability, which was developed to have content validity and found to have high interobserver and intraobserver agreement.

Glenohumeral joint instability is extremely common. With regard to primary anterior shoulder dislocations, the incidence is between 8.2 and 23.9 per 100000 person-years, with an estimated prevalence of $1.7 \% .{ }^{1-3}$ Whereas these estimates seem high, they actually underestimate the true nature of instability, as they do not include subluxation events or instability in other directions. Despite the widespread nature of glenohumeral joint instability, the definition of this condition is not clear and there is no consensus on how this disorder should be classified.

Historically, the medical literature regarding instability has a number of flaws. First, most studies in the literature are procedure-based and not condition-based. An example is the landmark 1980 paper by Neer and Foster ${ }^{4}$ on 'multidirectional' instability. In that study, the authors included patients with different features of instability-yet the patients had in common the same operation, an inferior capsular shift (table 1). When a paper is procedure based it may include a heterogenic population that will produce confusion both regarding the definition of the condition and whether the procedure described would be helpful for a particular patient in your office. Instead, papers should be condition-based, in which a population of patients with a specific, well-defined constellation of features is collected and two treatments are compared.

As a result of these historical problems, we do not have clear definitions for glenohumeral joint instability in the literature and papers tend to use a pot-pouri of descriptive terms (voluntary, traumatic unidirectional Bankart lesion treated with surgery, unidirectional, multidirectional, bidirectional, traumatic, atraumatic, microtraumatic, etc). This problem leads to heterogeneity in the literature making comparisons of different treatments difficult and meta-analyses nearly impossible.

This confusion has been highlighted by McFarland et al, ${ }^{5}$ who compared four different classification systems for patients with instability
Table 1 Features of Neer and Foster's multidirectional instability population

\begin{tabular}{ll}
\hline Feature & $\begin{array}{l}\text { No with symptom } \\
\text { (N=40) for entire } \\
\text { population }\end{array}$ \\
\hline Solitary episode & 0 \\
More than one episode & 40 \\
Traumatic origin & 29 \\
Atraumatic origin & 7 \\
Direction anterior & 29 \\
Direction inferior & 40 \\
Direction posterior & 29 \\
Subluxation event & 38 \\
Dislocation event & 2 \\
Hyperlaxity & 17 \\
Presence of Bankart lesion & 5
\end{tabular}

The frequency with which different features of instability are found in Neer and Foster's classic 1980 paper on multidirectional instability—all had in common the same inferior capsular shift operation.

and found great variation, particularly with regard to multidirectional instability, leading the editors of the Journal of Bone and Joint Surgery to opine that the article by McFarland et al ${ }^{5}$ was a "...provocative call to action", and "Until the criteria for diagnosis are clearly defined, investigators will be unable to contribute in a compelling way to understand the condition since they cannot know whether studies are comparing 'apples and oranges'." This confusion in how instability is defined was also demonstrated by Chahal et al, ${ }^{7}$ who found that physicians had poor agreement when asked to classify clinical scenarios of glenohumeral joint instability. These works provide evidence that we need better ways of defining and classifying glenohumeral joint instability.

\section{DEFINITIONS OF INSTABILITY}

Before a disorder is classified, it must be defined very clearly. For example, does a pitcher with a dead-arm feeling when throwing have 'instability'? Does a patient with a posterior labral tear with pain, but not sensation of the joint slipping or a feeling of looseness-have 'instability'? Historically, many experts have offered different definitions for this common malady. In 1992, many of the North American shoulder experts met in Vail, Colorado, to help define and determine the state of the art for many shoulder conditions. ${ }^{8}$ Interestingly, these experts offered a variety of definitions for glenohumeral joint instability (figure 1). In light of these different perspectives, how can a consensus be reached? The two common themes in these definitions are symptoms and translation. We know that many patients may have symptoms (especially pain) without instability. We also know that laxity exists in many 
"...the inability to maintain the humeral head centered in the glenoid fossa" Overview and Directions of Future Research. The Shoulder: a Balance of Mobility and Stability, Matsen FA, Fu FH, Hawkins RJ eds, AAOS, Rosemont, Illinios, 1993, p. 3.

"Instability is an abnormal limit of motion associated with a functional deficit (dynamic instability) or subluxation/dislocation (static or dynamic instability). A dynamically unstable configuration of load state would be one in which an increment of load will cause pain and inability to continue the function; in other words, "functionally buckling". A statically unstable configuration and load state would be the one in which an incremental increase in load leads to a large displacement with subsequent subluxation or dislocation." Lew WD, Lewis JL, Craig EV. Stabilization by Capsule, Ligaments, and Labrum: Stability at the Extremes of Motion. The Shoulder: a Balance of Mobility and Stability, Matsen FA, Fu FH, Hawkins RJ eds, AAOS, Rosemont, Illinios, 1993, p. 84.

"Glenohumeral instability can be defined as pain associated with the loss of shoulder function as a result of excessive translation of the humeral head on the glenoid fossa" Friedman RJ. Glenohumeral Capsulorraphy. The Shoulder: a Balance of Mobility and Stability, Matsen FA, Fu FH, Hawkins RJ eds, AAOS, Rosemont, Illinios, 1993, p. 446.

"This is a condition of a joint characterized by an abnormal increased amount of mobility secondary to injury of the ligaments, capsule, bone etc; when applied to the shoulder, instability typically is used to describe a clinical condition characterized by physical signs and related patients symptoms of increased or excessive displacement of the glenohumeral joint." Rodkey WG, Noble JS, Hintermeister RA. Laboratatory Methods of Evaluating the Shoulder. The Shoulder: a Balance of Mobility and Stability, Matsen FA, Fu FH, Hawkins RJ eds, AAOS, Rosemont, Illinios, 1993, p.570

"Instability is a clinical diagnosis manifest as excessive translation of the humeral head on the glenoid occurring during active shoulder rotation in association with symptoms" Warner JJP, Boardman ND III. Anatomy, Biomechanics, and Pathophysiology of Glenohumeral Instability. In The Unstable Shoulder. RF Warren, EV Craig, and DW Altchek eds, Lippencott-Raven, Philadelphia, 1999, p 51-2.

"Instability is defined as excessive symptomatic translation of the humeral head relative to the glenoid articular surface during active motion" Allen AA. Clinical Evaluation of the Unstable Shoulder. In The Unstable Shoulder. RF Warren, EV Craig, and DW Altchek eds, Lippencott-Raven, Philadelphia, 1999, $p 93$. patients without symptoms, and that some patients can sublux their shoulders without symptoms. Therefore, these two elements must be found together to define instability-patients must have discomfort and a feeling of looseness, slipping, or the shoulder 'going out' to meet the definition of instability.

\section{CLASSIFICATION OF INSTABILITY}

Once the condition is clearly defined, its features can be studied and categorised into meaningful classification systems. The classification of instability would help us in many ways. Instability classification systems should alert us to specific anatomical features we can expect to see with a specific type of instability, they should give us information regarding the natural history and prognosis of a type of instability, and they ultimately should offer recommendations regarding treatment.

A variety of classification systems have been proposed for shoulder instability. ${ }^{9-25}$ The authors of these papers developed their instability classification system after careful thought, yet the variation is remarkable. Although there is some agreement regarding the features that are included (figure 2), there is also a great amount of discordance, and different features are included in different classification systems. Interestingly, none of these systems has undergone reliability testing or validation. It is not surprising that there is no standard method to classify instability and that studies have demonstrated that patients may be given different diagnoses when different methods of classifying instability are used, ${ }^{5}$ and that physicians have poor agreement in how they describe the same patient. ${ }^{7}$

\section{THE FEDS SYSTEM FOR CLASSIFYING GLENOHUMERAL JOINT INSTABILITY}

At our institution we undertook a systematic approach to develop a method to classify shoulder instability - the FEDS classification. ${ }^{26}$ We performed a systematic review of the literature to identify proposed classification systems, and determined which features of instability were used most commonly (figure 2). Of all of the features of instability used by the 


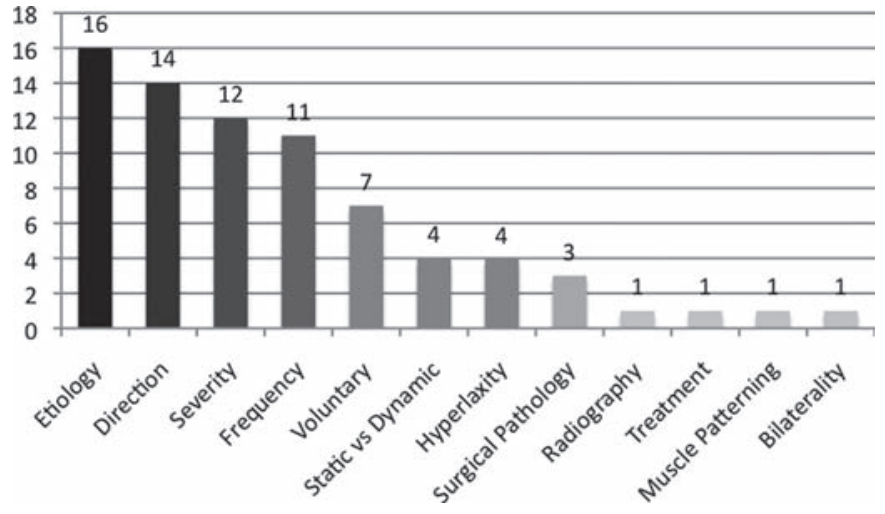

Figure 2 Different criteria used by different authors in proposed classification systems of glenohumeral joint instability. A systematic review identified 18 different proposed classification systems of glenohumeral instability. The frequency with which different features are used is presented. Aetiology, direction, severity and frequency were the most commonly used features (from Allen, ${ }^{9}$ Cole and Warner, ${ }^{10}$ Cofield and Irving, ${ }^{11}$ Galinat and Warren $1990,{ }^{12}$ Gerber and Nyffeler, ${ }^{13}$ Joseph et al, ${ }^{14}$ Lewis et al, ${ }^{15}$ Maruyama et al, ${ }_{1}^{16}$ Nebelung, ${ }^{17}$ Ozkan et al, ${ }^{18}$ Pollock and Flatow, ${ }^{19}$ Protzman, ${ }^{20}$ Rockwood, ${ }^{21}$

Schneeberger and Gerber, ${ }^{22}$ Silliman and Hawkins, ${ }^{23}$ Thomas and Matsen, ${ }^{24}$ Wirth and Rockwood ${ }^{25}$ ).

authors, four were seen in more than $50 \%$ of the proposed classification systems: frequency, aetiology, direction and severity. Interestingly, these features were reflected in the results of a survey of the membership of the American Shoulder and Elbow Surgeons. Features rated as extremely important in making the diagnosis of instability included: (1) the patient's history and physical examination in the office; (2) a history of trauma; (3) patients demonstrating the position of the arm that reproduces symptoms; (4) reproduction of symptoms using provocative testing and (5) determining the direction of the instability by physical examination. Interestingly the radiographic features and examination under anaesthesia were not rated as extremely important.

This information then led to the development of the FEDS classification for instability. Fortunately, the features of instability deemed most important (frequency, aetiology, direction and severity) could be obtained by history and physical examination. After a meeting of experts, the FEDS system was developed (figure 3).

\section{Frequency}

Frequency is an indirect measure of the severity of pathology, and is helpful in determining the approach to the patient. We purposefully chose the number of episodes over the course of 1 year due to the seasonal nature of sports. In considering frequency, the FEDS system has three levels. Solitary = one episode. It is likely that many patients with one episode will be managed non-operatively. Occasional $=$ two to five episodes. The patient with a few episodes may have a very different type of instability to the patient with more than five episodes per year, which would be described as frequent.

\section{Aetiology}

Most classification systems consider aetiology to be important and argue that patients with a history of trauma are managed differently to those without. While repetitive loading leading to symptoms has been described in the past as 'microtraumatic' or 'subtle' instability, we purposefully chose not to include this as a separate class (see below). Most athletes with shoulder trouble do not have instability by the definition above (a feeling that the shoulder is slipping, loose or going out)-rather they present with pain. As such, they would not be defined as having glenohumeral instability. Some athletes could have a feeling that their shoulder is loose without a specific injury. This group would fall under the atraumatic group.

\section{Direction}

Nearly every classification system includes the direction of the instability. In the FEDS classification, we rely on the history and the patient's perception of the instability. Patients may be able to tell you the direction of their instability, or may be able to tell you which position of the arm will reproduce their symptoms (hand behind the head for anterior, carrying a briefcase for inferior, pushing on something in front of the body for posterior). If the patient cannot tell the physician, the effect of provocative tests (translation anterior, inferior, posterior or apprehension test, sulcus sign, jerk test) will help determine the direction. We purposefully decided to eliminate the concept of 'multidirectional instability', and instead focused on the primary direction of symptoms when describing the direction of the instability (see below).

\section{Severity}

The severity of the instability is another criterion that comes from the history and is another indirect measure of the severity of the pathology. Patients can have subluxations or dislocations. It may be difficult to distinguish between these; however, in general subluxations will autoreduce, whereas dislocations will not. Therefore, the question for the patient: "Did you need, or have you ever needed help getting your shoulder back in?" is a yes or no question. It serves as a differentiating point, for the severity of the injury may help us gain some insight into the pathology.

\section{TESTING OF THE FEDS SYSTEM}

The FEDS classification system was assessed for interobserver and intraobserver agreement in a population of 48 patients with instability defined by answering yes to the question: "Do you feel like your shoulder is slipping, unstable, loose, or falling out of place?" These patients completed a survey, as did one of six sports medicine fellowship trained specialists. Patients returned after a minimum of 2 weeks and completed the survey again, as did the original treating physician and a second physician. Intraobserver agreement for the FEDS system was $84-97 \%$ with $k$ ranging from 0.69 (substantial) to 0.87 (almost perfect). Interobserver agreement was $82-90 \%$, with $k$ ranging from 0.44 (moderate) to 0.76 (substantial). ${ }^{26}$

\section{DISCUSSION}

The FEDS classification system is highly dependent upon the history and therefore the patient's perception of the disorder. Of all of the possible criteria for classifying instability, frequency, aetiology, severity and even direction can be obtained by questioning patients. Other criteria used historically to classify glenohumeral joint instability are physician derived, and include findings of examination under anaesthesia, examination and physician judgement as to the presence of a volitional nature to the instability, examination and physician judgement as to the presence of hyperlaxity, radiographic findings and pathological findings from surgery. 
FREQUENCY-The patient is asked, "How many episodes have you had in the last year?"

Solitary - ‘1 Episode'

Occasional- '2 -5 Episodes'

Frequent - '>5 Episodes'

ETIOLOGY - The patient is asked, 'Did you have an injury to cause this?'

Traumatic - 'Yes'

Atraumatic - 'No'

DIRECTION - The patient is asked, ' What direction does the shoulder go out most of the time?'

\section{Anterior- 'Out the Front' \\ Inferior- ' Out the Bottom' \\ Posterior- 'Out the Back'}

The direction is confirmed at the time of the physical examination using provocative tests. During translation testing, the physician asks, which one of the following directions most closely reproduces your symptoms, and then translates anterior, inferior, and posterior. To confirm, the physician may ask which one of these tests most closely reproduces your symptoms: and the anterior apprehension test, the sulcus test, and the posterior jerk test is performed. With the history and physical examination using provocative tests, the patient should be able to distinguish and identify the primary direction of his or her instability.

SEVERITY-The patient is asked,'Have you ever needed help getting the shoulder back in joint?'

Subluxation- 'No'

Dislocation - 'Yes'
Figure 3 The FEDS classification for instability.
In general, physician-derived criteria are less valuable, as the findings during examination are highly dependent upon the skill and experience of the examiner. As such, examination findings are highly subjective and interrater/intrarater reliability is poor. ${ }^{27-29}$ In addition, radiographic findings are dependent upon the presence of imaging tools, which may not be available in some parts of the world, and are likely to change as technology improves with time. Using pathological findings from surgery as a criterion is not ideal as it precludes making a diagnosis until surgery is performed. This would prevent the assignment of patients into groups for trials of non-operative therapy.

Interestingly, provocative physical examination tests designed to reproduce the patient's symptoms for instability, including the anterior apprehension test, the sulcus sign and load and shift tests, have been found to be sensitive, specific, and have high predictive values, with reasonable interexaminer reliability. ${ }^{30-33}$ Therefore, these features of the evaluation of the patient with instability can be useful. In the FEDS system, they are used in a comparative fashion to identify the primary direction of instability by finding which provocative test is most uncomfortable, or most closely reproduces the patient's symptoms.

\section{The problem with 'multidirectional' instability}

With regard to the direction of the instability, we intentionally focused on determining the primary direction of the instability and did not use the 'multidirectional' concept. We did this for the following reasons: (1) The term 'multidirectional instability' has been used by different authors to mean different things,, 5 and as a result the literature is very confusing. ${ }^{6} 1434$ (2) Neer and Foster ${ }^{4}$ in 1980 originally described the condition of multidirectional instability as having the sine qua non-feature of an increased sulcus sign, which would equate to having primary inferior symptoms in the proposed classification. (3) It could be argued that every form of shoulder instability could have excessive translations in multiple planes, as biomechanical and clinical research suggests that the capsule of the glenohumeral joint behaves as a circle and that injuries are unlikely to produce damage in only one part of the capsule. ${ }^{35-39}$ As a result, the concept of 'multidirectional' instability is flawed and it is unlikely to have a clear agreedupon definition among clinicians-as such we argue for its elimination, substituting the primary direction to describe the direction of instability instead.

\section{The problem with 'subtle instability'}

Rowe ${ }^{40}$ described the 'dead arm' syndrome in 1987. While some patients described a sense of shoulder looseness, many did not. Rowe considered all to have instability and performed instability surgery as a treatment. The problem is, as Rowe noted, pain is not specific for instability. Many of Rowe's patients had "signs and symptoms of bursitis, biceps tendonitis, nerve impingement, cervical spine referred pain and thoracic outlet syndrome. ${ }^{140}$ As such, it is not clear if these patients truly had instability.

Jobe $e t$ al ${ }^{41}$ in 1989 used 'subtle instability' to describe the athlete with shoulder pain. In this condition the patient may not have symptoms of the shoulder subluxing or dislocating, yet excessive laxity in the capsule presumably leads to other 
pathologies and other symptoms such as pain. Jobe used an instability operation to treat these patients and reported good success.

We would argue that the term 'subtle instability' is a poor choice, and that perhaps 'excessive laxity' would have been better. Again we refer to the concept that symptoms of instability are required for the diagnosis of instability. As such, patients with the constellation of findings that are seen in the athlete with the painful shoulder do not belong in the diagnosis of instability unless the athlete has symptoms of the arm having episodes of being loose. We would argue that as our understanding of the pathomechanics of the thrower's shoulder develops, a unique system for classifying different grades of pathology in the painful shoulder of the athlete will evolve.

\section{The problems with 'voluntary' instability}

In 1973, Rowe et al42 published a series of patients with 'voluntary instability'. After performing psychological testing on these patients, Rowe brought attention to the fact that patients with secondary gain age or psychiatric pathology did poorly with treatment. Those with voluntary instability who tested normally on psychological testing did well with treatment. ${ }^{42}$ It would seem that there are two types of voluntary instability that can be distinguished by psychological testing. However, because physicians do not perform psychological testing on their patients, this concept has led to a great amount of confusion in the literature. A number of other descriptors for this condition exist in the literature, including 'habitual instability'43 (which has erroneously included voluntary and involuntary by some authors) ${ }^{44}$ and 'involuntary positional instability'. ${ }^{45}$ These definitions are confused in the literature and it is difficult to distinguish which patients may have psychological issues. ${ }^{15}$ As a result, we do not believe the term 'voluntary instability' is particularly helpful in a classification of glenohumeral instability. Instead, we would consider using this concept as a qualifier for the descriptions above, and change the terminology to 'demonstrable instability' to describe patients who can demonstrate their instability but have no psychological or secondary gain issues, and 'volitional instability' for those patients who have the desire for their shoulder to sublux or dislocate. We would also recommend that researchers consider psychological testing on these patients to validate their classification of patients when studying such patients.

\section{CONCLUSION}

Any classification system must meet the following criteria: (1) it must be simple and easy to use; (2) it must accurately describe different types of instability with exclusion so that there is no ambiguity with assignment of patients; (3) it must have high reliability; (4) it should reflect the patient's perception of the disorder so that the integrity of outcome measures can be preserved; (5) it should be useful in predicting the natural history and possible treatment options.

It is clear that current methods of classifying glenohumeral joint instability have led to much confusion in the literature and do not meet these criteria. We suggest that a standard definition of instability should be used, and should include symptoms of the shoulder slipping, falling out, subluxing or dislocating. Using this definition, patients who are diagnosed as having glenohumeral joint instability should be classified using a system that is clear and contains the important features of the disorder. We propose that clinicians and researchers or investigators consider using the FEDS system, which by its development has content validity and has been shown to have high intraobserver and interobserver agreement. Once patients are clearly classified, only then can comparative studies of treatments be undertaken.

Provenance and peer review Not commissioned; not externally peer reviewed. Competing interest None.

\section{REFERENCES}

1. Hovelius L. Incidence of shoulder dislocation in Sweden. Clin Orthop Relat Res 1982;166:127-31.

2. Nordqvist A, Petersson CJ. Incidence and causes of shoulder girdle injuries in an urban population. J Shoulder Elbow Surg 1995;4:107-12.

3. Simonet WT, Melton LJ III, Cofield RH, et al. Incidence of anterior shoulder dislocation in Olmsted County, Minnesota. Clin Orthop Relat Res 1984;186:186-91.

4. Neer CS II, Foster CR. Inferior capsular shift for involuntary inferior and multidirectional instability of the shoulder. A preliminary report. J Bone Joint Surg Am 1980;62:897-908.

5. McFarland EG, Kim TK, Park HB, et al. The effect of variation in definition on the diagnosis of multidirectional instability of the shoulder. J Bone Joint Surg Am 2003;85-A:2138-44.

6. Richards RR. The diagnostic definition of multidirectional instability of the shoulder: searching for direction. J Bone Joint Surg Am 2003;85-A:2145-6.

7. Chahal J, Kassiri K, Dion A, et al. Diagnostic and treatment differences among experienced shoulder surgeons for instability conditions of the shoulder. Clin $J$ Sport Med 2007;17:5-9.

8. Matsen FA, Fu FH, Hawkins RJ, eds. The shoulder: a balance of mobility and stability. Rosemont, Illinois, USA: American Academy of Orthopaedic Surgeons, 1993.

9. Allen AA. Clinical evaluation of the unstable shoulder. In: Warren RE, Craig EV, Altchek DW, eds. The unstable shoulder. Philadelphia, Pennsylvania, USA: Lippincott-Raven, 1999:93-106.

10. Cole BJ, Warner JJP. Anatomy, biomechanics, and pathophysiology of glenohumeral instability. In: lannotti JP, Williams GR, eds. Disorders of the shoulder. Philadelphia, Pennsylvania, USA: Lippincott Williams and Wilkins, 1999:207-32.

11. Cofield RH, Irving JF. Evaluation and classification of shoulder instability. With special reference to examination under anesthesia. Clin Orthop Relat Res 1987:223:32-43.

12. Galinat BJ, Warren RF. Shoulder: trauma and related instability. In: Orthopedic knowledge update 3. Park Ridge, Illinois: American Academy of Orthopedic Surgeons, 1990. p 303.

13. Gerber C, Nyffeler RW. Classification of glenohumeral joint instability. Clin Orthop Relat Res 2002;400:65-76.

14. Joseph TA, Williams JS Jr, Brems JJ. Laser capsulorrhaphy for multidirectional instability of the shoulder. An outcomes study and proposed classification system. Am J Sports Med 2003;31:26-35.

15. Lewis A, Kitamura T, Bayler JIL. The classification of shoulder instability: new light through old windows! Curr Orthop 2004;18:97-108.

16. Maruyama K, Sano S, Saito K, et al. Traumatic instability-voluntarism classification for glenohumeral instability. J Shoulder Elbow Surg 1995:4:194-8.

17. Nebelung W. [Classification of recurrent shoulder joint instability]. Z Orthop Ihre Grenzgeb 2001;139:M84-7.

18. Ozkan M, Ekin A, Bölükbasi S, et al. [Shoulder instability: classification and methods of clinical examination]. Acta Orthop Traumatol Turc 2005;39 (Suppl 1):14-23.

19. Pollock RG, Flatow EL. Classification and evaluation. In: Bigliani LU, ed. The unstable shoulder. Rosemont, Illinois, USA: American Academy of Orthopaedic Surgeons, 1996:25-36.

20. Protzman RR. Anterior instability of the shoulder. J Bone Joint Surg Am 1980;62:909-18.

21. Rockwood CA. Subluxation of the shoulder: the classification diagnosis, and treatment. Orthop Trans 1979;4:306.

22. Schneeberger AG, Gerber C. [Classification and therapy of the unstable shoulder]. Ther Umsch 1998:55:187-91.

23. Silliman JF, Hawkins RJ. Classification and physical diagnosis of instability of the shoulder. Clin Orthop Relat Res 1993;291:7-19.

24. Thomas SC, Matsen FA III. An approach to the repair of avulsion of the glenohumeral ligaments in the management of traumatic anterior glenohumeral instability. J Bone Joint Surg Am 1989;71:506-13.

25. Wirth MA, Rockwood CA. Traumatic glenohumeral instability: pathology and pathogenesis. In: Matsen FA, Fu FH, Hawkins RJ, eds. The shoulder: a balance of mobility and stability. Rosemont, Illinois, USA: American Academy of Orthopaedic Surgeons, 1993:279-305. 
26. Kuhn JE, Holmes TT, Throckmorton TW, et al. Development and reliability testing of a system for classifying glenohumeral joint instability. American Academy of Orthopaedic Surgeons 75th Annual Meeting, 6 March 2008, San Francisco, California.

27. Ellenbecker TS, Mattalino AJ, Elam E, et al. Quantification of anterior translation of the humeral head in the throwing shoulder. Manual assessment versus stress radiography. Am J Sports Med 2000;28:161-7.

28. Ellenbecker TS, Bailie DS, Mattalino AJ, et al. Intrarater and interrater reliability of a manual technique to assess anterior humeral head translation of the glenohumeral joint. J Shoulder Elbow Surg 2002;11:470-5.

29. Levy AS, Lintner S, Kenter K, et al. Intra- and interobserver reproducibility of the shoulder laxity examination. Am J Sports Med 1999;27:460-3.

30. Tzannes A, Murrell GA. Clinical examination of the unstable shoulder. Sports Med 2002;32:447-57.

31. Tzannes A, Paxinos A, Callanan M, et al. An assessment of the interexamine reliability of tests for shoulder instability. J Shoulder Elbow Surg 2004;13:18-23.

32. Speer KP, Hannafin JA, Altchek DW, et al. An evaluation of the shoulder relocation test. Am J Sports Med 1994;22:177-83.

33. Lo IK, Nonweiler B, Woolfrey M, et al. An evaluation of the apprehension, relocation, and surprise tests for anterior shoulder instability. Am J Sports Med 2004;32:301-7.

34. Lo IK, Bishop JY, Miniaci A, et al. Multidirectional instability: surgical decision making. Instr Course Lect 2004;53:565-72.

35. Warner JJP, Boardman ND. Anatomy, biomechanics, and pathophysiology of glenohumeral instability. In: Warren RF, Craig EV, Altchek DW, eds. The unstable shoulder. Philadelphia: Lippincott-Raven, 1999:51-76.
36. Ovesen J, Nielsen S. Anterior and posterior shoulder instability. A cadaver study. Acta Orthop Scand 1986;57:324-7.

37. Tillander B Norlin R. Intraoperative measurement of shoulder translation $J$ Shoulder Elbow Surg 2001;10:358-64.

38. Warner JJ, Micheli LJ, Arslanian LE, et al. Patterns of flexibility, laxity, and strength in normal shoulders and shoulders with instability and impingement. Am J Sports Med 1990;18:366-75.

39. Yoldas EA, Faber KJ, Hawkins RJ. Translation of the glenohumeral joint in patients with multidirectional and posterior instability: awake examination versus examination under anesthesia. J Shoulder Elbow Surg 2001:10:416-20.

40. Rowe CR. Recurrent transient anterior subluxation of the shoulder. The "dead arm" syndrome. Clin Orthop Relat Res 1987;223:11-19.

41. Jobe FW, Kvitne RS, Giangarra CE. Shoulder pain in the overhand or throwing athlete. The relationship of anterior instability and rotator cuff impingement. Orthop Rev 1989;18:963-75.

42. Rowe CR, Pierce DS, Clark JG. Voluntary dislocation of the shoulder. A preliminary report on a clinical, electromyographic, and psychiatric study of twenty-six patients. J Bone Joint Surg Am 1973;55:445-60.

43. Kessel L, Bayley I. Clinical disorders of the shoulder, 2nd edn. Edinburgh, UK: Churchill Livingstone, 1986:189-97.

44. Huber $\mathbf{H}$, Gerber $\mathrm{C}$. Voluntary subluxation of the shoulder in children. A long-term follow-up study of 36 shoulders. J Bone Joint Surg Br 1994;76:118-22.

45. Takwale VJ, Calvert P, Rattue H. Involuntary positional instability of the shoulder in adolescents and young adults. Is there any benefit from treatment? J Bone Joint Surg Br 2000:82:719-23. 